\title{
Testing the sexual imagination hypothesis for gender differences in response to infidelity
}

Tsukasa Kato

\begin{abstract}
Background: Evolutionary psychologists hypothesized that men are more upset by sexual infidelity than women are, whereas women are more upset by emotional infidelity than men are. On the other hand, the sexual imagination hypothesis states that gender differences in infidelity responses are derived from explicit men's sexual imagery. Based on the latter hypothesis, we hypothesized that although men would report being more distressed by sexual infidelity than women who were not in a committed relationship (NCR), no gender difference would be reported in a committed relationship (CR).
\end{abstract}

Findings: These two hypotheses were tested with 598 participants in a CR and 1,643 participants in a NCR. No significant gender difference was found sexual infidelity response in the CR group ( $d=0.008$, a power of .956), whereas men were more upset than women about sexual infidelity in the NCR group. Moreover, a significant interaction between gender and infidelity type was found in the NCR, whereas no significant interaction between gender and infidelity type was observed in the CR group (partial $\eta^{2}=0.005$, a power of .943).

Conclusions: Our findings supported the sexual imagination hypothesis but were inconsistent with the EJM hypothesis.

Keywords: Sexual infidelity, Jealousy, Evolutionary psychology, Sexual imagination hypothesis, Gender difference

\section{Findings}

The sexual imagination hypothesis regarding gender differences in infidelity responses was tested by comparing individuals who were in a committed relationship (CR) with individuals who were not in a committed relationship (NCR). Our results supported the sexual imagination hypothesis.

\section{Background}

According to some evolutionary psychologists [1-3], men are more upset by a mate's sexual infidelity than women are, whereas women are more upset by a mate's emotional infidelity than men are. In the ancestral history of humans, such sex differences are ascribed to the need for reproductive fitness. Ancestral males could not be certain that a putative offspring was their own. For males, a mate's sexual infidelity poses the risk of investing resources unknowingly in the offspring of rivals. Therefore, males are more vigilant about their mate's sexual infidelity to prevent this. However, ancestral

Correspondence: mtsukasa@hotmail.com

Department of Social Psychology, Toyo University, 5-28-20 Hakusan Bunkyo-ku, Tokyo 112-8606, Japan females incur no such risk because they do not doubt that the putative offspring is their own. However, females face a different risk, that is, emotional infidelity, wherein their mates invest resources in the offspring of their rivals. This evolutionary view is referred to as the sex-specific evolved jealousy mechanism (EJM). Based on the EJM hypothesis, Buss et al. [1] asked survey participants to imagine their partner's infidelity using brief scenarios and then identify the more distressing of the two types of infidelity. Gender differences in responses to a partner's infidelity were reported; men were more upset by sexual infidelity than women were, and women were more upset by emotional infidelity than men were. Such differences in infidelity responses have been found repeatedly (for reviews, see $[4,5]$ ).

However, some researchers have questioned the validity of the EJM hypothesis due to contradictory findings [6-10] and alternative interpretations to account for the gender differences in infidelity response. The sexual imagination hypothesis $[10,11]$ states that gender differences in infidelity responses are derived from men's explicit sexual imagery $[10,11]$. According to this hypothesis, men and women will not differ in responses to a partner's 
sexual infidelity, if women too can imagine sexual infidelity as vividly and realistically as men. However, men are more upset by a partner's sexual infidelity than women are, because men are more likely than women to imagine explicit details (for a review, see [10]). For example, Geer and McGlone [12] found that men were faster and more accurate in recognition tasks involving erotic sentences than women are. Schützwohl and Koch [13] showed that men recalled more cues to sexual infidelity than women did. In addition, data from men and women who have actually experienced infidelity showed no significant gender differences in sexual infidelity reactions [14-18]. Moreover, Kato [10] found that there were no significant gender differences in jealousy when sexual infidelity was imagined in a laboratory using vivid infidelity scenarios and photographs to induce detailed explicit imagery of a partner's infidelity.

In the present study, gender differences in response to a partner's sexual infidelity were tested in two groups: those who were currently in a committed relationship (CR) and those who were not in a committed relationship (NCR). Based on the sexual imagination hypothesis, we hypothesized that although men in a NCR group would report being more distressed than women because men are better able to imagine sexual infidelity than women, no gender difference would be reported in a CR group. Previous studies [7,19-21] have reported that relationship status was a predictor of gender differences in jealousy. Some $[7,19]$ of these studies found that women in a CR were more distressed or upset over sexual infidelity than women in a NCR. Some studies $[7,19,21]$ showed, among married individuals or those in a CR, no significant gender differences in sexual infidelity reactions. In addition, previous studies [19] using Buss's [1] infidelity scenarios showed that individuals in a NCR reported more difficulty imagining aspects of infidelity than those in a CR. For example, our analysis of Becker et al.'s [19] data revealed that the difference between individuals in a CR and those in a NCR in imagining infidelity was large effect size (Cohen's $d=1.14$ ). However, previous studies that showed no significant gender differences in jealousy about sexual infidelity did not take into account the Type II or beta $(\beta)$ error probability of falsely retaining an incorrect null hypothesis with relatively small sample sizes (about from 200 to 350). We also tested the EJM hypothesis using responses to emotional infidelity as well as sexual infidelity in CR and NCR groups.

\section{Methods}

\section{Participants and procedure}

Participants were 2,241 college students (1,449 women and 792 men; $M=19.48$ years, $\mathrm{SD}=1.52$ ) enrolled in introductory psychology classes. All participants were born in Japan and identified their ethnicity as Japanese.
Participants who were currently in a committed relationship were 404 women and 194 men $(M=19.80$ years, $\mathrm{SD}=1.63$ ); in the present study, we refer to this group as the CR group. Participants who were not in a committed relationship were 1,045 women and 598 men $(M=$ 19.37 years, $\mathrm{SD}=1.47$ ); we refer to this group as the NCR group. Twenty-one potential participants did not provide their gender or relationship status; therefore, they were not included in the study. No one in either group had been married. A committed relationship in the present study referred to a serious and potentially long-lasting romantic committed heterosexual relationship, which did not include casual dating. After giving informed consent, they completed a set of questionnaires in small groups supervised by research assistants. They received course credit for their participation. All procedures were in accordance with the ethical standards of the responsible committee on human experimentation (institutional and national) and with the Helsinki Declaration of 1975, as revised in 2000; this project was approved by the Institutional Ethics Committee of Toyo University.

\section{Measures}

Participants were asked questions about their reactions to infidelity scenarios by Buss et al. [1]. The scenarios were as follows: Please think of a serious committed romantic relationship that you have had in the past, that you currently have, or that you would like to have. Imagine your partner enjoying passionate sexual intercourse with that other person. Imagine your partner forming a deep emotional attachment to that person. Participants were instructed to rate how upset or distressed they would be by each type of infidelity on a 6-point Likerttype scale, ranging from 1 (not at all upset or distressed) to 6 (extremely upset or distressed). Jealousy was assessed using continuous measures for two reasons. First, evolutionary psychologists have employed such scales to demonstrate sex differences in jealousy (for reviews see [5,9]). Second, there are serious methodological issues with hypothetical forced-choice findings [9]; for example, they are incapable of independently assessing sexual and emotional jealousy. We assessed sexual infidelity independently in order to test the sexual imagination hypothesis. The two infidelity scenarios were presented in random order. No significant effects of presentation order for sexual infidelity $(t(2239)=0.16, p=.88)$ and emotional infidelity $(t(2239)=0.81, p=.42)$ scores were found.

These infidelity scenarios were originally written in English and translated into Japanese using the back translation method by Kato [10]. Using this Japanese version revealed a similar pattern to Buss et al.'s main findings [1], Kato [10] suggested that his Japanese version was a credible measure to use. 


\section{Data analysis}

A 2 (gender) $\times 2$ (group: CR vs. NCR groups) $\times 2$ (infidelity type) analysis of variance (ANOVA) was conducted to test the sexual imagination hypothesis and the EJM hypothesis. In order to test the former hypothesis, planned comparisons for an interaction between gender and group on sexual infidelity response scores were conducted. In addition, planned comparisons for an interaction between gender and infidelity type were conducted for each infidelity type to test the latter hypothesis.

According to Sagarin [22], evidence of an interaction between gender and type of infidelity would demonstrate the EJM hypothesis when participants used continuous rating scales to estimate their distress over the two types of infidelity, which need not show a cross-over pattern (see [9]). However, Harris [9] stated that slopes for men and women should be in opposite directions or show a cross-over interaction in order to document the EJM hypothesis. Sagarin supported the interpretation of EJMbased gender differences in jealousy, whereas Harris is skeptical about the EJM hypothesis. We tested the evolutionary explanation for gender differences using Sagarin's [22] approach; that is, we examined interactions between gender and infidelity type in the CR and NCR groups. If the EJM hypothesis, both groups should show a significant interaction between gender and infidelity type at minimum.

\section{Results}

Means and standard deviations of responses to sexual and emotional infidelity are shown in Table 1 . The $2 \times$ $2 \times 2$ ANOVA revealed a significant three-way interaction at $p<.05, F(1,2237)=10.12$, partial $\eta^{2}=0.005$.

\section{Sexual imagination hypothesis}

A significant interaction between gender and group with sexual infidelity responses was found at $p<.05: F(1,2237)=$ 4.11, partial $\eta^{2}=0.002$; but no significant interaction with emotional infidelity responses was found at $p<.05$ (see Figure 1): $F(1,2237)=1.39$, partial $\eta^{2}=0.001$. Planned

Table 1 Means and standard deviations of responses to sexual and emotional infidelity

\begin{tabular}{lrrrrrr}
\hline & \multicolumn{3}{c}{ Men } & & \multicolumn{3}{c}{ Women } \\
\hline Group & $N$ & $M$ & SD & $N$ & $M$ & SD \\
Sexual infidelity & & & & & & \\
CR & 194 & 4.68 & 1.31 & 404 & 4.67 & 1.20 \\
NCR & 598 & 4.64 & 1.17 & 1045 & 4.40 & 1.03
\end{tabular}

Emotional infidelity

$\begin{array}{lrrrrrr}\text { CR } & 194 & 4.63 & 1.20 & 404 & 4.79 & 1.04 \\ \text { NCR } & 598 & 4.40 & 1.10 & 1045 & 4.68 & 0.92\end{array}$

Note. $\mathrm{CR}=$ group who currently in a committed relationship. $\mathrm{NCR}=$ group who not in a committed relationship.

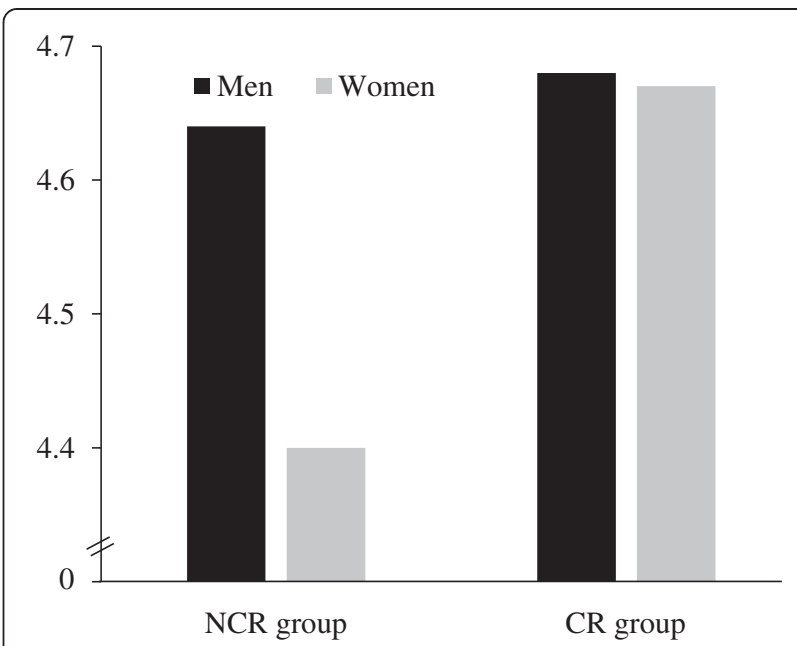

Figure 1 Means of Responses to sexual infidelity in the group who currently in a committed relationship (CR) and the group who not in a committed relationship (NCR).

comparisons for the interaction revealed that women in the $\mathrm{CR}$ group $(M=4.67, \mathrm{SD}=1.20)$ reported being more upset or distressed than women in the NCR group $(M=$ 4.40, $\mathrm{SD}=1.03), 95 \%$ confidence interval (CI) [0.152, $0.400]$ for the difference scores, $d=0.27$. Men in the NCR group $(M=4.64, \mathrm{SD}=1.17)$ reported being more upset or distressed than women in the NCR group $(M=4.40$, $\mathrm{SD}=1.03), 95 \%$ CI $[0.129,0.347], d=0.24$, but there was no such significant gender difference in the CR group $(p=.947,95 \%$ CI $[-0.205,0.219], d=0.008)$. In addition, a post-hoc power analysis for the gender differences in the CR using the G*Power program version 3.1.7 [23] revealed a power of .956 , based on an effect size of Cohen's $d=$ 0.008 and a total sample size of $N=598$. The $95 \% \mathrm{CI}$ $[-0.205,0.219]$ for the difference scores was narrow. These results were consistent with our expectations.

\section{EJM hypothesis}

A significant interaction between gender and infidelity type in the NCR group was found $(F(1,1641)=87.34$, partial $\left.\eta^{2}=0.051\right)$, but no significant interaction in the CR group was found at $p<.05(F(1,596)=2.79$, partial $\left.\eta^{2}=0.005\right)$. The NCR group result was consistent with the EJM hypothesis, but the CR group result was inconsistent with the EJM hypothesis. In addition, a post-hoc power analysis for the interaction in the CR group revealed a power of .943 , based on an effect size of partial $\eta^{2}=0.005$ and a total sample size of $N=2,241$.

\section{Discussion}

Planned comparisons for the interaction between gender and group revealed that men reported being significantly more upset or distressed in sexual infidelity responses than did women in the NCR group, whereas there was 
no significant gender difference in the CR group and a small effect size (Cohen's $d=0.008$ ) and narrow 95\% CI for the difference scores. The power analysis for the gender difference in the CR group showed a high value (power $=.956$ ), indicating the high probability that its null hypothesis will be rejected given that it is in fact false. That is, the type II error probability is low. Our findings were consistent with previous studies [7,19,21] and supported the validity of the sexual imagination hypothesis.

In addition, the interaction between gender and infidelity type in the NCR group was significant, whereas it was not significant in the CR group with a small effect size (partial $\eta^{2}=0.005$ ). The CR group result was inconsistent with the evolutionary prediction for gender differences in jealousy. The power $(1-\beta)$ was .943 for the CR group interaction, indicating that the type II error probability is low. If the evolutionary explanation is valid, gender differences in jealousy should be observed among individuals in a CR rather than those in a NCR, because the evolutionary interpretation explains gender differences in jealousy for human couples but not for noncoupled males and females. The results in our sample did not support the EJM hypothesis. Although our data cannot categorically deny the possibility of the evolutionary explanation for gender differences in responses to a partner's infidelity, our findings indicate that the EJM hypothesis cannot provide an explanation for gender differences in infidelity in our sample.

\section{Conclusions}

We found no significant gender differences in response to a partner's sexual infidelity the CR group. In addition, no significant interaction between gender and infidelity type was found in the CR group. Our findings supported the sexual imagination hypothesis, but were inconsistent with the EJM hypothesis.

\footnotetext{
Abbreviations

CR: individuals who were in a committed relationship; EMJ: sex-specific evolved jealousy mechanism; NCR: individuals who were not in a committed relationship.
}

\section{Competing interests}

The author declares that he has no competing interests.

\section{Acknowledgements}

There was not anyone who contributed towards this article. In addition, the author did not receive any funding for this article.

Received: 26 May 2014 Accepted: 25 November 2014

Published: 29 November 2014

\section{References}

1. Buss DM, Larsen RJ, Westen D, Semmelroth J: Sex differences in jealousy: evolution, physiology, and psychology. Psychol Sci 1992, 3:251-255.

2. Daly M, Wilson M, Weghorst SJ: Male sexual jealousy. Ethol Sociobio/ 1982, $3: 11-27$.
3. Symons D: The Evolution of Human Sexuality. New York: Oxford University Press; 1979

4. Easton JA, Schipper LD, Shackelford TK: Morbid jealousy from an evolutionary psychological perspective. Evol Hum Behav 2007, 28:399-402.

5. Sagarin BJ, Martin AL, Coutinho SA, Edlund JE, Patel L, Skowronski JJ, Zengel B: Sex differences in jealousy: a meta-analytic examination. Evol Hum Behav 2012, 33:595-614.

6. DeSteno D, Bartlett MY, Braverman J, Salovey P: Sex differences in jealousy: evolutionary mechanism or artifact of measurement? J Pers Soc Psychol 2002, 83:1103-1116.

7. Guadagno RE, Sagarin BJ: Sex differences in jealousy: an evolutionary perspective on online infidelity. J App/ Soc Psychol 2010, 40:2636-2655.

8. Harris CR: A review of sex differences in sexual jealousy, including self-report data, psychophysiological responses, interpersonal violence, and morbid jealousy. Pers Soc Psychol Rev 2003, 7:102-128.

9. Harris CR: Male and female jealousy, still more similar than different: reply to Sagarin (2005). Pers Soc Psychol Rev 2005, 9:76-86.

10. Kato T: A reconsideration of sex differences in response to sexual and emotional infidelity. Arch Sex Behav 2014, 43:1281-1288.

11. Harris CR: Psychophysiological responses to imagined infidelity: the specific innate modular view of jealousy reconsidered. J Pers Soc Psychol 2000, 78:1082-1091.

12. Geer $\mathrm{JH}, \mathrm{McGlone} M \mathrm{~S}$ : Sex differences in memory for erotica. Cogn Emot 1990, 4:71-78

13. Schützwohl A, Koch S: Sex differences in jealousy: the recall of cues to sexual and emotional infidelity in personally more and less threatening context conditions. Evol Hum Behav 2004, 25:249-257.

14. Berman MI, Frazier PA: Relationship power and betrayal experience as predictors of reactions to infidelity. Pers Soc Psychol Bull 2005, 31:1617-1627.

15. Buunk B: Jealousy in sexually open marriages. Alternative Lifestyles 1981, 4:357-372.

16. Harris CR: Sexual and romantic jealousy in heterosexual and homosexual adults. Psychol Sci 2002, 13:7-12.

17. Harris CR: Factors associated with jealousy over real and imagined infidelity: an examination of the social-cognitive and evolutionary psychology perspectives. Psychol Women Q 2003, 27:319-329.

18. Tagler MJ: Sex differences in jealousy: comparing the influence of previous infidelity among college students and adults. Soc Psychol Pers Sci 2010, 1:353-360.

19. Becker DV, Sagarin BJ, Guadagno RE, Millevoi A, Nicastle LD: When the sexes need not differ: emotional responses to the sexual and emotional aspects of infidelity. Pers Relationsh 2004, 11:529-538.

20. Burchell JL, Ward J: Sex drive, attachment style, relationship status and previous infidelity as predictors of sex differences in romantic jealousy. Pers Individ Dif 2011, 51:657-661.

21. Voracek M: Marital status as a candidate moderator variable of male-female differences in sexual jealousy: the need for representative population samples. Psychol Rep 2001, 88:553-566.

22. Sagarin BJ: Reconsidering evolved sex differences in jealousy: comment on Harris (2003). Pers Soc Psychol Rev 2005, 9:62-75.

23. Faul F, Erdfelder $E$, Lang A, Buchner A: G*Power 3: a flexible statistical power analysis program for the social, behavioral, and biomedical sciences. Behav Res Methods 2007, 39:175-191.

\section{doi:10.1186/1756-0500-7-860}

Cite this article as: Kato: Testing the sexual imagination hypothesis for gender differences in response to infidelity. BMC Research Notes 2014 7:860. 